\title{
Adrenal Ganglioneuroma with Multifocal Retroperitoneal Extension: A Challenging Diagnosis
}

\author{
Marco Oderda ${ }^{1}$, Elena Cattaneo ${ }^{1}$, Francesco Soria ${ }^{1}$, Antonella Barreca ${ }^{2}$, \\ Luigi Chiusa ${ }^{2}$, Bruno Morelli ${ }^{1}$, Andrea Zitella ${ }^{1}$, and Paolo Gontero ${ }^{1, *}$ \\ ${ }^{1}$ Department of Urology-1, Molinette Hospital, University of Turin, Italy; ${ }^{2}$ Department \\ of Biomedical Sciences and Human Oncology, University of Turin, Italy \\ E-mail: paolo.gontero@unito.it
}

Received April 21, 2011; Revised July 27, 2011; Accepted July 29, 2011; Published August 16, 2011

\begin{abstract}
A ganglioneuroma (GN) is the rarest and most benign of the neuroblastic tumors and originates from neural crest cells wherever sympathetic nervous tissue exists, such as in the retroperitoneum and adrenal gland. The diagnosis can be very challenging, given the rarity and asymptomatic presentation of this neoplasia, and can be achieved only by means of histological evaluation. Although benign, a few cases of metastatic GNs have been reported in the literature. The prognosis, however, seems to be excellent after surgical resection. We describe a rare case of multifocal retroperitoneal GN, diagnosed incidentally in a 46-year-old woman, with para-aortic and adrenal localizations. After intraoperative pathological diagnosis was made, complete excision of all the visible masses was performed. The postoperative period was uneventful and she was recurrence free 3 months after surgery. To our knowledge, this is the first case report of a multifocal retroperitoneal GN. Among the broad differential diagnoses of adrenal incidentalomas, an adrenal location of neuroblastic tumors should not be forgotten.
\end{abstract}

KEYWORDS: neuroganglioma, adrenal, sympathetic tissue, diagnosis, therapy

\section{INTRODUCTION}

The differential diagnosis of an adrenal incidentaloma is broad. Although rare, an adrenal location of neuroblastic tumors should be included among the diagnostic hypotheses. A ganglioneuroma (GN) is the rarest and most benign of the neuroblastic tumors[1,2]. It originates from the neural crest cells and arises wherever sympathetic tissue exists, including the adrenal medulla[1,2]. A retroperitoneal GN is typically asymptomatic and its diagnosis incidental. The diagnosis is not assisted by imaging studies and can be obtained only by means of histological evaluation[1,10]. Therefore, given the rarity of this neoplasia, the diagnosis can be very challenging. Although benign, a few cases of metastatic GNs have been described in the literature[2,8,9]. The prognosis, however, seems to be excellent after complete surgical resection[ $[1,2,8,10]$. We describe the first case ever reported of a multifocal retroperitoneal GN. 


\section{CASE REPORT}

During a routine physical examination, a left adrenal mass was incidentally discovered on ultrasound (US) in a 46-year-old female. Abdominal computed tomography (CT) revealed an oval-shaped, hypodense neoformation with well-defined margins, in contact with the left adrenal gland, located between the pancreatic tail and the upper pole of the left kidney (Fig. 1). The mass was $36 \times 56 \mathrm{~mm}$ in size and demonstrated no visible contrast enhancement (Fig. 2). There was no evidence of lymphadenopathy within the abdomen or pelvis. All laboratory exams were within normal ranges, including serum cortisol, androgen hormones, and urine levels of catecholamines and their metabolites. Surgical removal of the mass was planned. During the exploration of the abdominal cavity, multiple para-aortic masses similar to enlarged lymph nodes were found, simulating lymphoma with adrenal gland involvement. The surgical specimen, sent for intraoperative pathological evaluation, consisted of an oval soft fragment, measuring $4 \times 3 \times 2 \mathrm{~cm}$. Frozen section analysis did not reveal any evidence of malignancy and suggested the diagnosis of a retroperitoneal extra-adrenal GN. On the basis of this finding, surgery proceeded successfully with the completion of left adrenalectomy and the excision of all the visible para-aortic masses.

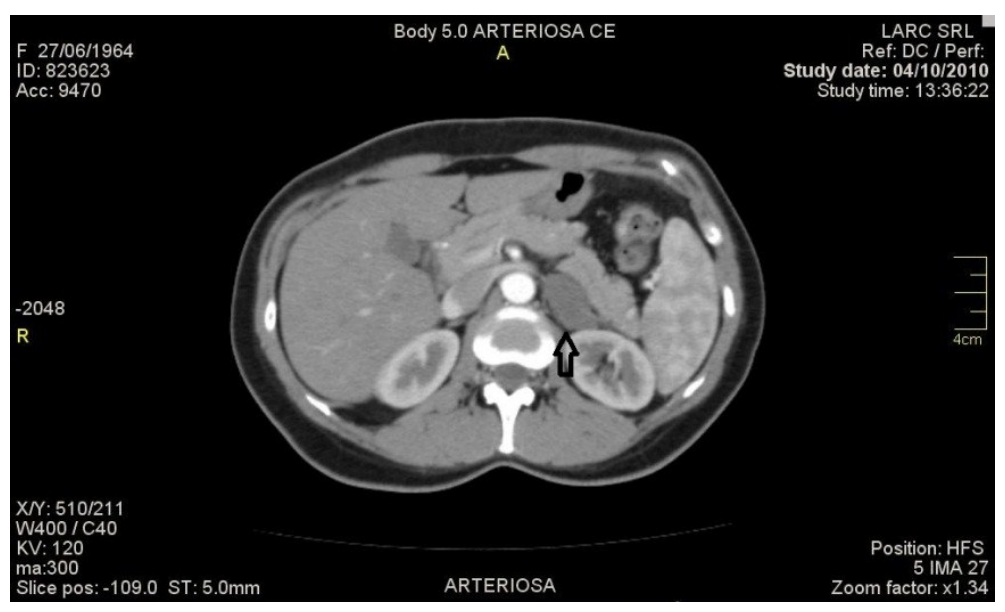

FIGURE 1. CT image: the arrow points to the left adrenal mass.

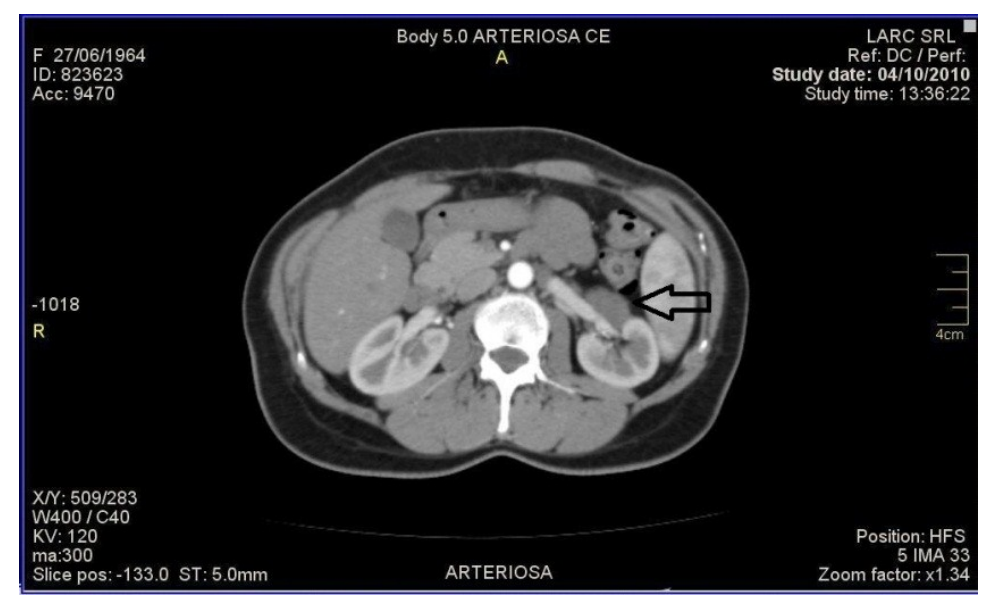

FIGURE 2. CT image: the arrow points to a mass next to the renal artery. Intraoperatively, this mass was detached from the previous one, demonstrating the multifocality of the GN. 
The definitive histological examination confirmed the diagnosis of GN for all the masses removed, without lymph node involvement. Two nodes in relation to the left adrenal gland, 5 and $3 \mathrm{~cm}$ in size, respectively, appeared well circumscribed and partly encapsulated. The para-aortic masses consisted of two nodes, 1.2 and $2 \mathrm{~cm}$ in size, respectively, the former well demarcated and adjacent to several lymph nodes with reactive hyperplasia and the latter with irregular borders. On cut section, all the surgical fragments were grayish with a whorled pattern. Histologic examination showed the presence of bundles of longitudinal and transversely oriented Schwann cells with an irregular fashion (Fig. 3A). Scattered through the Schwannian background were relatively mature ganglion cells (Fig. 3B), usually arranged in small clusters or nests. Ganglion cells had voluminous bright pink cytoplasms and one to three nuclei, which showed mild atypia. There were also a lot of blood vessels and small areas of calcification. Furthermore, myxoid degeneration of stroma could be appreciated (Fig. 3C). The adrenal gland was adjacent to the tumor, but the two entities were clearly separated (Fig. 3D). The Schwann and ganglion cells resulted diffusely S100 positive (Fig. 3E). The proliferative index, measured by means of ki-67 expression, was $<1 \%$ (Fig. 3F).
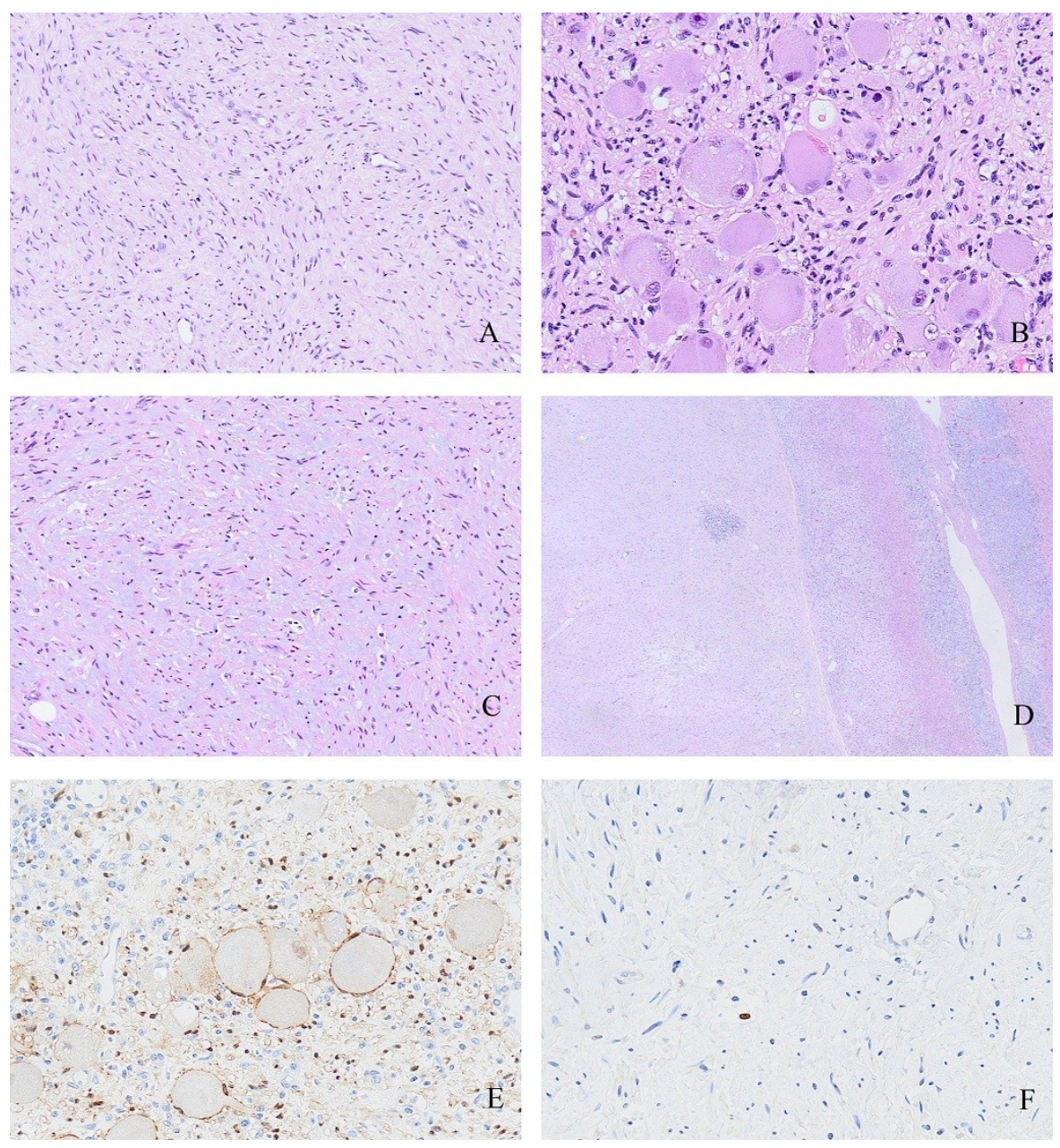

FIGURE 3. Histologic sections of the extra-adrenal retroperitoneal GN. The tumor was composed of nerve fibers (A) and mature ganglion cells (B) without any malignant features. (C) Myxoid degeneration of neuromatous stroma. (D) The tumor was clearly separated from the adrenal gland. (E) Immunopositivity for S100 was observed in ganglion and Schwann cells. (F) Ki-67 expression was $<1 \%$. 
The postoperative course was uneventful and the patient was discharged on the fifth postoperative day. Thoracic CT scan was performed in order to rule out mediastinal or thoracic involvement. Three months after surgery, the patient was well and had no signs of tumor recurrence.

\section{DISCUSSION}

Ganglioneuromas, ganglioneuroblastomas, and neuroblastomas are neuroblastic tumors of neural crest origin and arise wherever sympathetic tissue exists. Ganglioneuroblastomas and neuroblastomas are immature neoplasms characterized by aggressive behavior, early occurrence, and poor prognosis[1]. On the contrary, GNs are the most benign and well differentiated of these tumors, composed entirely of mature sympathetic ganglion cells, Schwannian stroma, fibrous tissue, and nerve fibers. By definition, GNs do not contain immature elements, such as neuroblasts, mitotic figures, or intermediate cells[2], thus predicting a favorable prognosis. GNs can arise de novo, or arise from maturing ganglioneuroblastomas or neuroblastomas, especially when treated with chemotherapy[3]. This kind of tumor is very rare and typical of infant age, although a recent review reported that retroperitoneal GNs actually appear in patients younger than 18 years in only one-third of the cases[4]. Several series showed mean age at diagnosis to fall within the $3^{\text {rd }}$ and the $4^{\text {th }}$ decade of life [5,6], which is concordant with our case. GNs arise in association with the sympathetic nervous tissue, more frequently in the posterior mediastinum $(41.5 \%)$ and in the retroperitoneum (37.5\%)[1]. Only $20-30 \%$ occur in the adrenal medulla[1], while other locations include the neck, spermatic cord, heart, bone, and intestine[1,7]. Although benign, GNs can exceptionally metastasize to distant sites; there are a few reports of metastases to regional lymph nodes[2,8], liver, spleen[9], and soft tissues[2]. These cases of metastatic GNs are believed to be the results of the spreading of neuroblastomas or ganglioneuroblastomas that subsequently matured to GNs. Pathologic diagnosis is essential in order to identify lymph node metastases; our case mimicked a metastatic GN with lymph node involvement, but pathologic diagnosis actually revealed only hyperplasia in para-aortic lymph nodes contiguous to GNs. The multifocality of presentation of this yet rare neoplasia is noteworthy; to our knowledge, in the literature, there are no reports of multifocal retroperitoneal GNs not associated with specific syndromes, such as multiple endocrine neoplasia type IIb (MEN IIb) and neurofibromatosis type 1 (NF1).

The clinical presentation of GNs is typically asymptomatic, given that most of these tumors are hormone silent and incidentally discovered during routine radiographic examination, as in our case[1,10]. Furthermore, retroperitoneal GNs tend to produce less symptoms than intraperitoneal GNs and they can reach huge dimensions, up to $25 \mathrm{~cm}[4,11]$, before being diagnosed. GNs, however, have been associated with a variety of hormonal secretions, such as catecholamines, vasoactive intestinal peptides, and testosterone[2,5]. It has been shown that catecholamine production is found most frequently[2]. Differential diagnosis with pheochromocytoma is necessary, bearing in mind the eventuality of a composite pheochromocytoma-GN, a mixed neuroendocrine-neural tumor that is composed of pheochromocytoma and other neural crest derivatives[12]. In our case, we also had to consider differential diagnosis with adrenal lymphoma, either primary or secondary[13]; the macroscopic intraoperative appearance of multiple para-aortic masses was suggestive of lymphoma and only histological evaluation allowed a correct diagnosis.

Imaging characteristics of GNs are not discriminating. US usually reveals a homogeneous, hypoecoic mass with well-defined borders[1,10]. On CT, GNs appear as hypodense and homogeneous, demonstrating slight to moderate contrast enhancement[1,10]. At magnetic resonance imaging, GNs show low signal intensity on T1-weighted images and heterogeneous high signal intensity on T2-weighted images[10]. None of these exams, however, allow differentiation among GNs and the other neuroblastic tumors or adrenal incidentalomas.

Concerning the management of GNs, a complete surgical resection is the preferred approach[1,10,11], although there is still no absolute consensus about the indications of surgery for adrenal masses. According to a 2004 National Institutes of Health State-of-the-Science statement[14], the 
generally accepted recommendation regarding nonfunctional adrenal masses is to excise asymptomatic lesions $>6 \mathrm{~cm}$, while close follow-up is indicated for asymptomatic lesions $<4 \mathrm{~cm}$. For lesions between 4 and $6 \mathrm{~cm}$, both surgery and follow-up can represent reasonable approaches. Our patient presented with a lesion in contact with the left adrenal gland, $<6 \mathrm{~cm}$. Nevertheless, in consideration of the unknown origin of the mass and the young age of the patient, we decided to proceed with the surgery in order to resect the visible lesion and to obtain a histological diagnosis.

Fine-needle biopsies are not recommended as a standard diagnostic procedure in patients with adrenal masses[14]. Furthermore, in our case, the option of a biopsy was discarded because the particular location of the tumor, adjacent to the aorta and the pancreatic tail, made the procedure too risky. Preoperative diagnosis of a retroperitoneal $\mathrm{GN}$ is often challenging and usually confirmed by histopathological findings after surgical excision of the neoplasia.

Prognosis after surgical resection seems to be excellent without further therapies, even in the few documented cases of metastatic disease[1,2,8,10]. A neoadjuvant therapy was described to reduce the size of the tumor, but was ineffective[15]. Local recurrences have been reported, so long-term follow-up is recommended after surgery[1]. Our patient was recurrence free at CT executed 3 months for surgery. Given the multifocality of the lesion, close follow-up will be recommended.

\section{REFERENCES}

1. Lonergan, G.F., Schwab, C.M., Suarez, E.S., and Carlson, C.L. (2002) Neuroblastoma, ganglioneuroblastoma and ganglioneuroma: radiologic-pathologic correlation. Radiographics 22, 911-934.

2. Geoerger, B., Hero, B., Harms, D., Grebe, J., Scheidhauer, K., and Berthold, F. (2001) Metabolic activity and clinical features of primary ganglioneuromas. Cancer 91, 1905-1913.

3. Ambros, I.M., Zellner, A., Roald, B., et al. (1996) Role of ploidy, chromosome 1p, and Schwann cells in the maturation of neuroblastoma. N. Engl. J. Med.334, 1505-1511.

4. Papavramidis, T.S., Michalopoulos, N., Karayannopoulou, G., Kesisoglou, I., Tzioufa, V., Raptou, G., and Papavramidis, S.T. (2009) Retroperitoneal ganglioneuroma in an adult patient: a case report and literature review of the last decade. South. Med. J. 102(10), 1065-1067.

5. Rondeau, G., Nolet, S., Latour, M., Braschi, S., Gaboury, L., Lacroix, A., Panzini, B., Arjane, P., Cohade, C., and Bourdeau, I. (2010) Clinical and biochemical features of seven adult adrenal ganglioneuromas. J. Clin. Endocrinol. Metab. 95(7), 3118-3125.

6. Johnson, G.L., Hruban, R.H., Marshall, F.F., and Fishman, E.K. (1997) Primary adrenal ganglioneuroma: CT findings in four patients. Am. J. Roentgenol. 169, 169-171.

7. Jain, M., Shubba, B.S., Sethi, S., Banga, V., and Bagga, D. (1999) Retroperitoneal ganglioneuroma: a report of a case diagnosed by fine-needle aspiration cytology, with review of the literature. Diagn. Cytopathol. 21, 194-196.

8. Srinivasan, R., Koliyadan, K.S., Krishand, G., and Bhat, S.S. (2007) Retroperitoneal ganglioneuroma with lymphnode metastasis: a case report. Indian J. Pathol. Microbiol. 50(1), 32-35.

9. Chen, G.H. and He, J.N. (1986) Malignant ganglioneuroma of adrenals with metastasis to liver and spleen. Chin. Med. J. 99(3), 261-263.

10. Erem, C., Ucuncu, O., Nuhoglu, I., Cinel, A., Cobanoglu, U., Demirel, A., Koc, E., Kocak, M., and Guvendi, G.F. (2009) Adrenal ganglioneuroma: report of a new case. Endocrine 35, 293-296.

11. Lai, M.C., Wang, C.C., Lin, W.C., Liu, K.L., and Huang, K.H. (2011) Huge adrenal ganglioneuroma. Urology 78(1), 58-59.

12. Mahajan, H., Lee, D., Sharma, R., Chin, P., Watt, W.H., McBride, G., and Bilous, M. (2010) Composite phaeochromocytoma-ganglioneuroma, an uncommon entity: report of two cases. Pathology 42(3), 295-298.

13. Zhou, L., Peng, W., Wang, C., Liu, X., Shen, Y., and Zhou, K. (2010) Primary adrenal lymphoma: radiological, pathological, clinical correlation. Eur. J. Radiol. [Epub ahead of print]

14. Mansmann, G., Lau, J., Balk, E., Rothberg, M., Miyachi, Y., and Bornstein, S.R. (2004) The clinically inapparent adrenal mass: update in diagnosis and management. Endocr. Rev. 25(2), 309-340.

15. Qureshi, S.S. and Medhi, S.S. (2008) Large adrenal ganglioneuroma with left inferior vena cava: implications for surgery. Pediatr. Surg. Int. 24, 455-457. 
This article should be cited as follows:

Oderda, M., Cattaneo, E., Soria, F., Barreca, A., Chiusa, L., Morelli, B., Zitella, A., and Gontero, P. (2011) Adrenal ganglioneuroma with multifocal retroperitoneal extension: a challenging diagnosis. TheScientificWorldJOURNAL: TSW Urology 11, 1548-1553. DOI 10.1100/tsw.2011.144. 


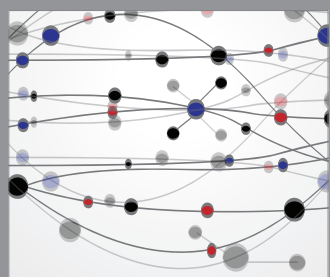

The Scientific World Journal
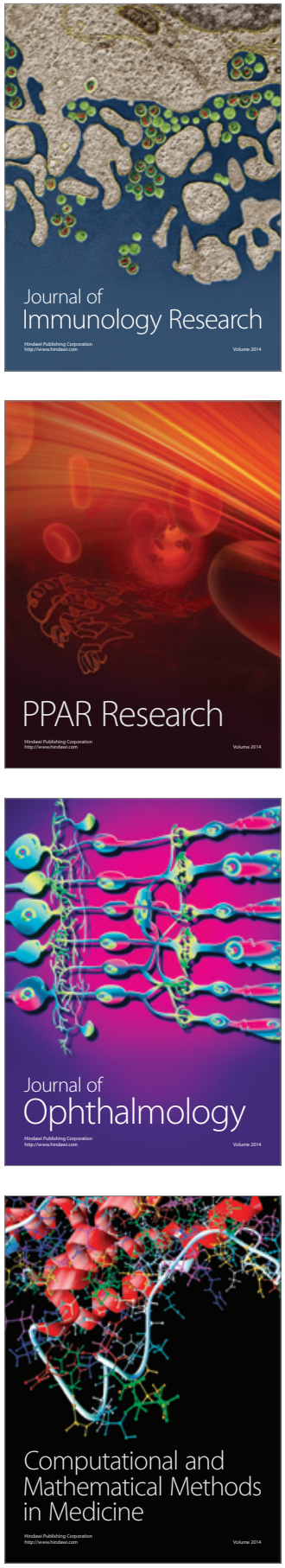

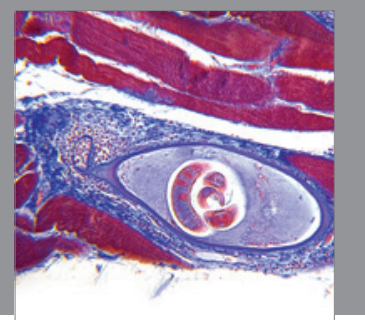

Gastroenterology

Research and Practice
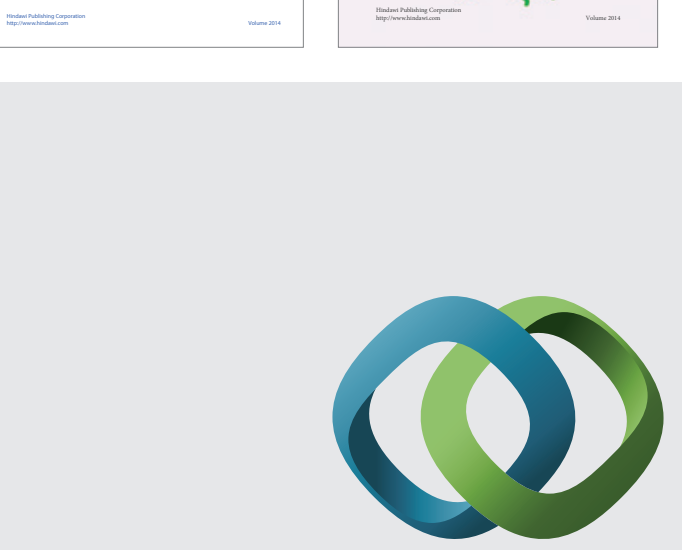

\section{Hindawi}

Submit your manuscripts at

http://www.hindawi.com
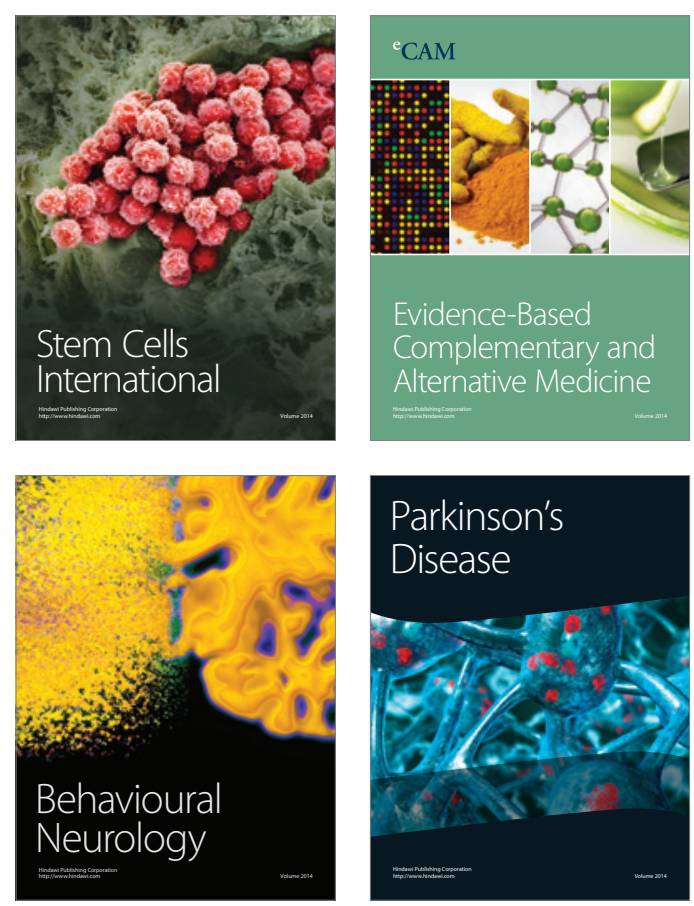

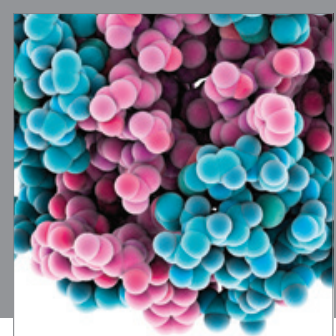

Journal of
Diabetes Research

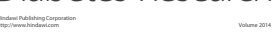

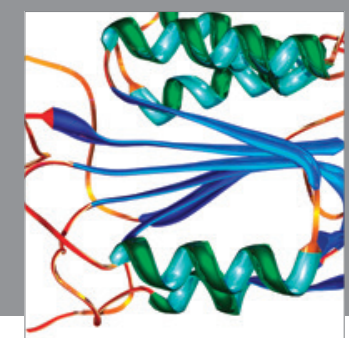

Disease Markers
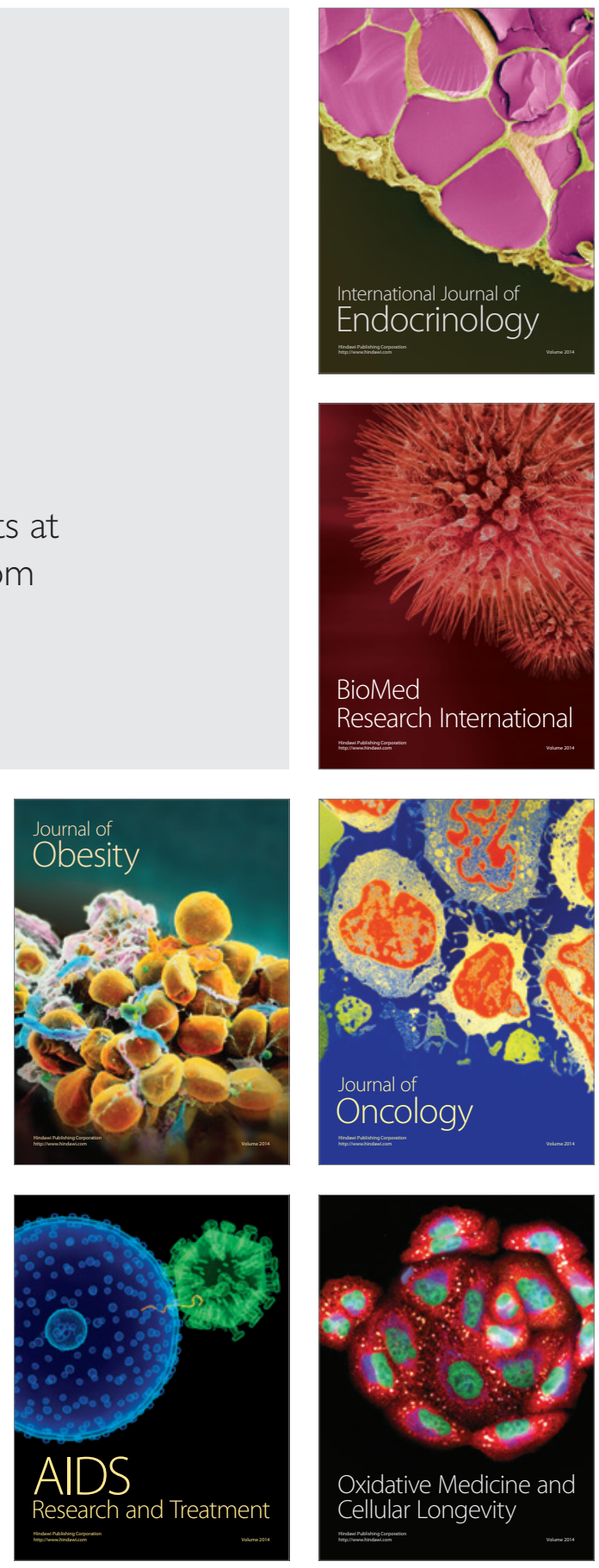